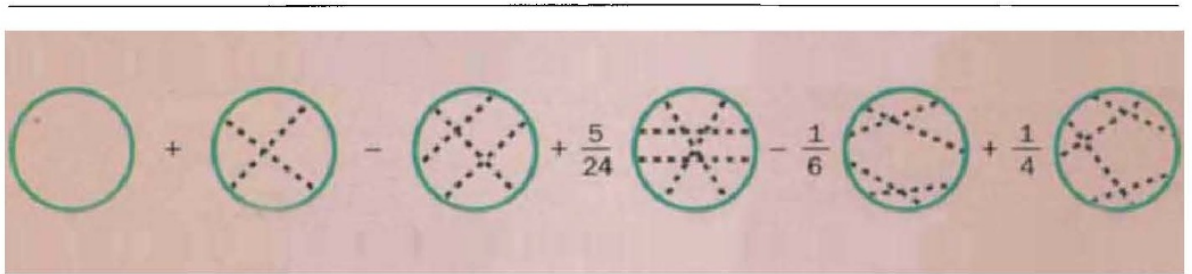

FIG. 3 The first six terms in an expansion of the trefoil knot in terms of chord diagrams. Each chord shows which parts of the circle are joined at double points (imagine shrinking the chord until the sides of the circle meet at its centre).

Similarly, a knot corresponds to a point in the space of all knots. But whereas the function space was three-dimensional in the quadratic case, the space of all knots has infinite dimensions, making it (to put it mildly) harder to visualize. In this space the different knot types are separated into chambers by walls, the walls consisting of knots that have a self-intersection or 'double point' (Figs $1 b$ and 2 show examples). The walls meet at 'corners' which correspond to knots with two self-intersections. This analysis of walls and corners then generalizes to knots with a greater number of self-intersections.

Vassiliev approached the study of these walls by studying numerical knot invariants (invariants that assign a number to every knot, and thus a single number to each 'chamber' described above). Such an invariant can be extended to knots with a single self-intersection - in other words, its value can be defined on the walls - by looking at the knots on either side of the wall. The number on the wall is simply the difference between the values on either side. Then this procedure can be extended to knots with two double points and so on. Vassiliev invariants are those invariants that are zero when extended to knots with a certain (large) number of double points. These invariants are quite simple to understand from certain viewpoints, and Birman and $\operatorname{Lin}^{6}$ proved that the Jones polynomial is built up from invariants of this type.

The other approach was from quantum physics where one considers the probability of events happening: the probability of 'observables' such as the position and momentum of particles taking certain values. Around the mid-1980s physicists started considering space-times with no notion of distance. Think of two points marked on a rubber sheet: it is impossible to define a distance between the points, as stretching the sheet would alter the distance. Without the notion of distance, position and momentum become meaningless, so one needs topological ways of describing the motion of particles: in fact the only sensible thing to consider as an observable is the knottedness of the particles' trajectories.

A tool known as the Feynman path integral can be used to calculate the probabilities of particles traversing knots in three-dimensional space-time, these probabilities depending only on the type of knot involved. A near-miraculous discovery was made by Witten ${ }^{7}$, a physicist at Princeton, when he was able to construct a model for space-time in which this probabilistic invariant generalized the Jones polynomial. However, although physicists happily use the Feynman integral (as it seems to give them sensible answers), it has never been properly defined mathematically. A more rigorous analysis of Witten's construction has been sorely needed.

And this is where Bar-Natan's work comes in. Working under Witten, BarNatan $^{2,8}$ used perturbative methods to expand the Witten integral as a sum of terms that are easily understood mathematically, rather like expanding $e^{x}$ as powers of $x$. This expansion is in terms of generalized Feynman diagrams (see Fig. 3 ); the integral describes all possible ways that the particle can behave, and each Feynman chord diagram corresponds to one of these types of behaviour. BarNatan has shown that the invariant derived from each chord diagram is a Vassiliev invariant, neatly uniting the two topological approaches.

Where next for the knot theorists? Kontsevich ${ }^{9}$ has constructed an integral that expands every knot as a sum of chord diagrams, and has proved that these methods give rise to all invariants of Vassiliev's type. His integral was originally impossible to calculate except for the simplest knots, but mathematicians are now finding ways of making it more calculable. So the latest results, although they have not answered every question, have certainly increased the arsenal with which knot theorists can attack their favourite tangles.

Simon Willerton is in the Department of Mathematics and Statistics, Edinburgh University, Mayfield Road, Edinburgh EH9 3JZ, UK.

1. Lord Kelvin Proc. R. Soc. Edinb. 6, 94-105; reprinted in Mathematical and Physical Papers Vol . 4, 1-12 (Cambridge Univ. Press, UK, 1910).

(Cambridge Univ. Press, UK, 1910).
Bar-Natan, D. Topology (in the press)

3. Kauffman, L. H. On Knots (Princeton Univ. Press, 1987)

4. Jones, V. F. R. Bull. Am. math. Soc. 12, 103-111 (1985)

5. Vassiliev, V. A. Complements of Discriminants of Smooth Maps: Topology and Applications (Trans. Math. Monogr. 98, American Mathematical Society, Providence, 1992).

6. Birman, J. \& Lin. X. S. Invent. Math. 111, 225-270 (1993).

7. Witten, E. Commun. math. Phys. 121, 351-399 (1989)

8. Bar-Natan, D. thesis, Princeton Univ. (1991).

9. Kontsevich, M. Adv. Sov. Math. 16 (2), 137-150 (1993).

\section{Less is more}

HOMOEOPATHY is a well-established fringe therapy. It offers paradoxical medicines whose effectiveness rises as their dose is reduced. The manufacturers seem curiously unconvinced by their own claim - they usually recommend a half dose for children, never the double dose required by the theory. But many formulations are so dilute that they are statistically unlikely to contain a single molecule of the active ingredient, so the precise dosage can hardly matter.

What chemical effects intensify with dilution? One candidate, says Daedalus, is electrochemical: the equilibrium potential on an electrode in solution. The Nernst equation asserts that this potential grows monotonically more negative as the concentration of the solution declines. Ultimately, zero concentration should give an infinite negative voltage. This extreme, of course, can never quite be reached. Even so, many ionic equilibria can be made so lopsided that, as in a homoeopathic remedy, there is only a small statistical chance of having even a single ion of a particular species in the solution. The Nernst negative potential is correspondingly great.

So Daedalus feels that homoeopathic remedies work, quite accidentally, by evoking Nernst potentials in the patient's tissues. They must contain impurities or trace components which release tiny concentrations of ions into the body. Common ions, such as sodium and calcium, would be swamped by the body's natural levels. Only rare and unusual ions could work: perhaps by acting on electrochemical organs such as nerves. Hence, of course, the popularity of homoeopathic treatment for the more nebulous and nervous afflictions.

DREADCO's electrochemists are now analysing homoeopathic remedies in search of traces of such nonbiochemicals as dysprosium, rhenium and various exotic organometallic ions. From the results, they will design a range of rational electrochemical remedies containing traces of ions not usually present in the body, and capable of setting up high Nernst potentials across its cell walls and nerve membranes.

DREADCO's 'homoelectric remedies' should compete strongly with the traditional varieties. Like these, their high dilution will make them very economical in raw materials. But as rational medicines, they will have an intelligible biochemical action. As a result, and in strong contrast to the suspicious innocuousness claimed for so many 'natural' remedies, they may have side effects.

David Jones 